〔Med. Entomol. Zool. Vol. 60 No. 1 p. 13-22 2009]

\title{
Characterization of trypsin-and chymotrypsin-like genes in the midgut of the tsetse fly Glossina morsitans morsitans (Diptera: Glossinidae)
}

\author{
Mikio Yoshiyama ${ }^{1)}$, Minoru Yamakawa ${ }^{2)}$, Yuichi Chigusa ${ }^{3)}$ \\ and Wendy C. GIBSON ${ }^{4)}$ \\ ${ }^{1)}$ Animal Breeding and Reproduction Research Team, National Institute of Livestock \\ and Grassland Science, Ikenodai 2, Tsukuba, Ibaraki, 305-0901 Japan \\ 2)Innate Immunity Laboratory, National Institute of Agrobiological Sciences, \\ Owashi 1-2, Tsukuba, Ibaraki, 305-8634 Japan \\ ${ }^{3}$ Department of Tropical Medicine and Parasitology, Dokkyo Medical University \\ School of Medicine, Mibu, Tochigi, 321-0293 Japan \\ ${ }^{4)}$ School of Biological Sciences, University of Bristol, Woodland Road, Bristol, BS8 IUG U.K.
}

(Received: 27 October 2008; Accepted: 26 December 2008)

\begin{abstract}
Putative serine protease genes including trypsin and chymotrypsin were identified in the tsetse fly Glossina morsitans morsitans during an EST project. Trypsin and chymotrypsin are major digestive enzymes involved in the parasite-host interactions of vector insects. In this study, two genes designated Gmmtry 1 and Gmmchy 1 were further characterized during blood digestion and in responses against parasite infection. Both Gmmtry 1 and Gmmchy 1 mRNA was expressed at high levels in the posterior region of the midgut. Gmmchyl transcript increased following an infectious blood meal suggesting chymotrypsin is regulated by the presence of the trypanosome during the early establishment of the parasite infection in the midgut. The possible roles of trypsin and chymotrypsin proteases in determining the refractoriness of the tsetse fly against trypanosomes is also discussed.
\end{abstract}

Key words: Tsetse fly, trypsin, chymotrypsin, trypanosome, digestive enzymes

\section{INTRODUCTION}

Tsetse flies are medically important vectors of protozoan trypanosomes that cause African trypanosomiasis in humans and animals (Aksoy et al., 2003). The WHO report that at least 100,000 new cases of human African trypanosomiasis occur each year and 60 million people are at risk in 37 countries (WHO, 2001). The agents of Sleeping sickness, African trypanosomes rely on the tsetse fly (Diptera: Glossinidae) for successful transmission. The mammalian bloodstream form of trypanosomes rapidly differentiates into a procyclic form in the tsetse midgut and the surfaces become covered with procyclic proteins (Ruepp et al., 1997). The majority of ingested parasites are completely eliminated around day 3 after infection. A small portion of surviving parasites continues to proliferate and establish infections in the anterior region of the midgut of the tsetse fly (Gibson and Bailey, 2003). After establishment in the midgut, the trypanosomes migrate forward to the mouthparts or salivary glands where they differentiate into an infectious metacyclic form (Vickerman et al., 1988). Therefore, the successful establishment of a trypanosome infection in the tsetse midgut greatly relies on the early stage around 3 days after infection. Tsetse flies 
are normally refractory to parasite transmission, even under ideal laboratory conditions infection rates are less than 20\% (Moloo et al., 1992, 1994). In addition, the infection rate of the fly populations in the field fails to exceed 5\% (Msangi et al., 1998).

Several factors, including sex of the tsetse fly, and presence of lectins, endosymbiotic bacteria and antimicrobial peptides play an important role in successful parasitic infection of the midgut (Maudlin and Ellis, 1985; Welburn and Maudlin, 1999; Hao et al., 2001; Hu and Aksoy, 2005; Hu and Aksoy, 2006).

Proteases are potentially important in the tsetse fly and trypanosome interactions. EP and GPEET procyclin, the major surface glycoproteins of procyclic forms of Trypanosoma brucei brucei, are truncated by serine proteases in the midgut of Glossina morsitans morsitans. Therefore, serine proteases have the potential to influence the tsetse fly and trypanosome interactions (Acosta-Serrano, 2001; Liniger et al., 2003, 2004).

An EST gene discovery project to identify putative protease genes including trypsin and chymotrypsin from the midgut of adult G. m. morsitans was conducted by Lehane et al. (2003). Previous studies reported that two serine protease genes of the tsetse fly are constitutively expressed (Yan et al., 2001). In addition, the lectin-trypsin complex was isolated from Glossina species and shown to stimulate the transformation of the bloodstream form trypanosome into the procyclic forms in vitro (Abubakar et al., 2006; Osir et al., 1995). Here we report further characterization of the trypsin and chymotrypsin genes from $G$. m. morsitans (named Gmmtry1, Gmmchy1), their expression profiles during blood digestion and responses against parasitic infection.

\section{Materials and Methods}

\section{Tsetse flies}

Glossina m. morsitans was originally es- tablished from puparia collected in Zimbabwe. The colony is kept in the insectary at Liverpool School of Tropical Medicine at $24 \pm 2{ }^{\circ} \mathrm{C}$ and $55-60 \%$ relative humidity. Flies receive defibrinated horse blood by an artificial membrane feeding system (Moloo, 1971). To survey expression profiles during parasite establishment, flies were fed blood containing $1 \times$ $10^{6} / \mathrm{m} l$ of bloodstream form $T$. b. bruce $i$ TSW 196. Flies fed on the infected meal were subsequently maintained on defibrinated sterile blood. Midguts were microscopically dissected at designated times.

\section{Gene contig analysis and Bioinformatics}

Genes encoding putative trypsin and chymotrypsin were retrieved from GeneDB (http://www. genedb. org / genedb/glossina/index.jsp), and a Blast search performed (http://www.ncbi.nlm. nih.gov/BLAST/). Signal peptide sequences and protein domains were searched for using SignalP 3.0 (http:// www.cbs.dtu.dk/services/SignalP/) and Pfam (http://www.sanger.ac.uk/Software $/$ Pfam/), respectively. Sequences were aligned by GENETYX-MAC ver 9.0 (Software Development).

\section{$R T-P C R$}

To determine tissue specific expression profiles, three parts of the midgut and the fat body were dissected from flies $24 \mathrm{~h}$ after feeding. Total RNA was isolated using the RNeasy kit (Qiagen) according to the manufacturer's instructions. After DNaseI treatment for $1 \mathrm{~h}$ at $37^{\circ} \mathrm{C}$, first strand cDNA was synthesized from $1 \mu \mathrm{g}$ of total RNA using a First Strand cDNA Synthesis kit (Promega) with oligodT primers. Gene specific primers, Gmmtry1QF; 5' TGC AGC TAC CAT GCC CAA GGG-3', Gmmtry1QR; 5'-ATT GCG CCT TGT AAG CAA TGG-3', Gmmchy 1QF; 5' -GGT TAC GTA CCC TGT ACG GCG-3', and Gmmchy1QR; 5'-CAA GAT GAA TCC TGC AAC ACA AAT-3' were used. The Gmm GAPDH clone (Gmm2682) from the 
Table 1. Trypsin and chymotrypsin-like genes identified from an EST library.

\begin{tabular}{|c|c|c|c|c|c|c|}
\hline Contig & Ortholog & Acc. No. & E value & Pfam & E value & $\begin{array}{c}\text { Sequence } \\
\text { length (bp) }\end{array}$ \\
\hline Gmm3293 & Dp GA14937-PA & EAL26142 & $3 e-45$ & Trypsin & $1.1 \mathrm{e}-06$ & 1,061 \\
\hline Gmm3216 & De delta trypsin & AAA 83243 & $2 e-38$ & Trypsin & $9 e-15$ & 1,093 \\
\hline Gmm3061 & Dm alpha trypsin & AAF58659 & $8 e-39$ & & & 979 \\
\hline Gmm3354 & Gm trypsin-like & AAF91346 & $1 \mathrm{e}-73$ & Trypsin & $4.9 \mathrm{e}-17$ & 896 \\
\hline Gmm3108 & Gm trypsin-like & AAF91346 & $2 \mathrm{e}-121$ & Trypsin & $1.4 \mathrm{e}-74$ & 1,140 \\
\hline Gmm3359 & Gm trypsin-like & AAF91346 & $2 \mathrm{e}-42$ & & & 1,016 \\
\hline Gmm2697 & Gm trypsin-like & AAF91346 & $4 e-59$ & & & 579 \\
\hline Gmm3340 & Gm trypsin-like & AAF91346 & $6 e-34$ & & & 915 \\
\hline Gmm3330 & Gm chymotryp. & AAF91345 & $1 e-66$ & & & 936 \\
\hline Gmm3362 & Gm chymotryp & AAF91345 & $5 e-39$ & & & 1,085 \\
\hline $\mathrm{Gmm} 3230$ & Gm chymotryp. & AAF 91345 & $3 e-121$ & Trypsin & $1.3 \mathrm{e}-38$ & 993 \\
\hline Gmm7528 & Dm GRAAL2 & CAC35209 & $7 e-54$ & Scav. recepter & $9.4 \mathrm{e}-25$ & 563 \\
\hline Gmm3310 & De Eta trypsin & AAA83237 & $1 e-21$ & & & 980 \\
\hline Gmm2574 & Gm Pro3 & AAN52278 & $3 e-56$ & & & 938 \\
\hline Gmm2542 & Gm Cathepsin B & AAK07477 & $1 e-89$ & & & 571 \\
\hline Gmm2539 & Gm Cathepsin B & AAK07477 & $3 e-95$ & papain & $3.3 \mathrm{e}-20$ & 584 \\
\hline Gmm7163 & Dm easter & AAF55170 & $6 e-61$ & & & 473 \\
\hline Gmm7162 & Dp GA18526 & EAL27739 & $5 e-37$ & & & 483 \\
\hline
\end{tabular}

EST was used as a reference gene. GmmGAPDH specific primers designed for PCR were GmmGAPDHF; 5' -CTC ATT CGT CGG TGT TCG ATG-3' and GmmGAPDHR; 5' -GCG TTG AAC AGC AAT ATT TCC CT- $3^{\prime}$.

\section{Real-time quantitative PCR}

First strand cDNA synthesis and gene specific primers were employed as described above. PCR reactions were performed in a final volume of $20 \mu \mathrm{l}$ with the LightCycler ${ }^{\circledR} 480$ system (Roche) using Fast Start Reaction Mix SYBR Green 1 (Roche). Cycling conditions were as follows: $95^{\circ} \mathrm{C}$ for $5 \mathrm{~min}$ followed by 40 cycles of $95^{\circ} \mathrm{C}$ for $10 \mathrm{~s}, 60^{\circ} \mathrm{C}$ for $5 \mathrm{~s}$ and $72^{\circ} \mathrm{C}$ for $10 \mathrm{~s}$. Serial dilutions of the $\mathrm{pGEM}^{\circledR}$-T Easy vector containing the GmmGAPDH gene were used as a standard curve. The expression levels were quantified by the second derivative maximum method of LightCycler Software (Roche). Transcript levels were normalized with the GAPDH gene as an internal control and the mean \pm standard deviations were calculated from the three independent experiments. The data were compared using the Student's $t$-test. Self-cleared flies were defined as those which had had a trypanosome-infected blood but which had no microscopically detectable trypanosomes in the midgut. Flies fed normal blood only were defined as controls.

\section{Trypsin and chymotrypsin enzyme assays}

Teneral G. $m$. morsitans were fed by artificial membrane. At designated times midguts were dissected and individually stored in $100 \mu \mathrm{l}$ of PBS. For trypsin activity, $1 \mathrm{mM} \quad \mathrm{N} \alpha$-benzoyl-DL-arginine p-nitroanilide (BApNA Sigma) was used. For chymotrypsin activity, $0.5 \mathrm{mM}$ of $\mathrm{N}$-succinyl-ala-ala-pro-phe-p-nitroanilide substrate (AAPF Sigma) was used. The substrates were dissolved in dimethylsulphoxide (DMSO) at $100 \mathrm{mM}$ Tris- $\mathrm{HCl}$ buffer ( $\mathrm{pH}$ 8.0) immediately prior to assay, 
(A)

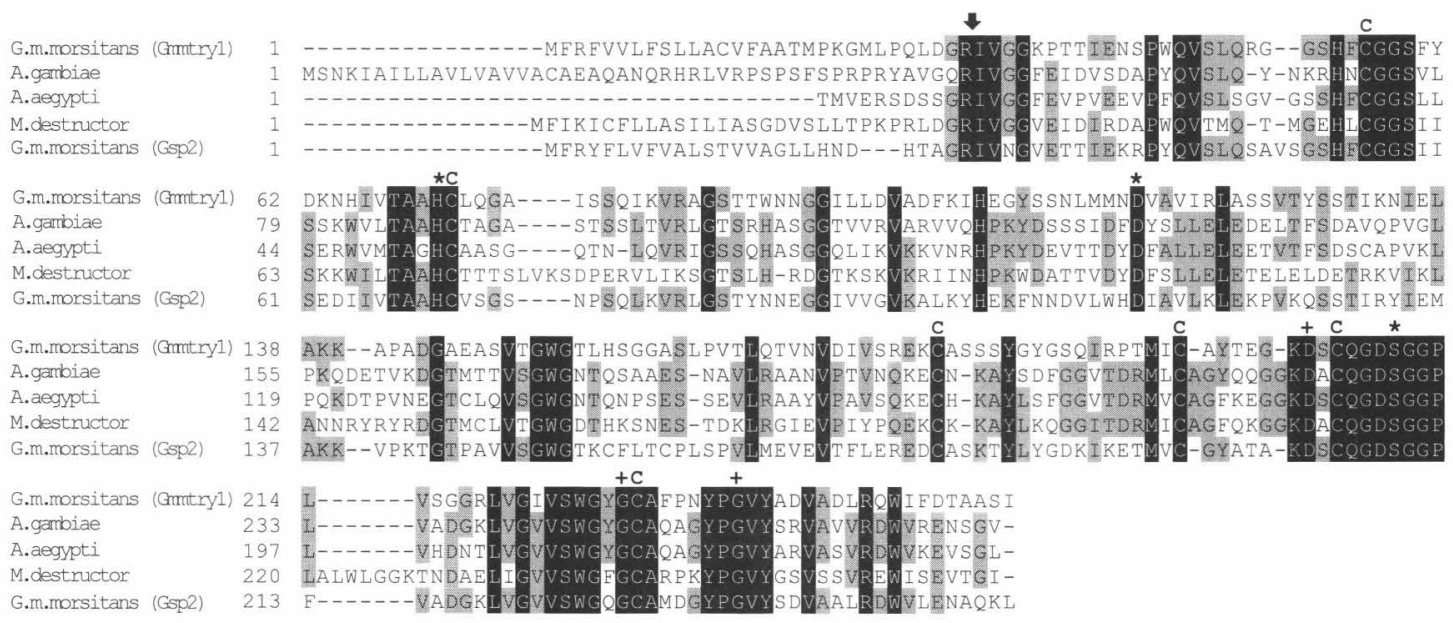

(B)

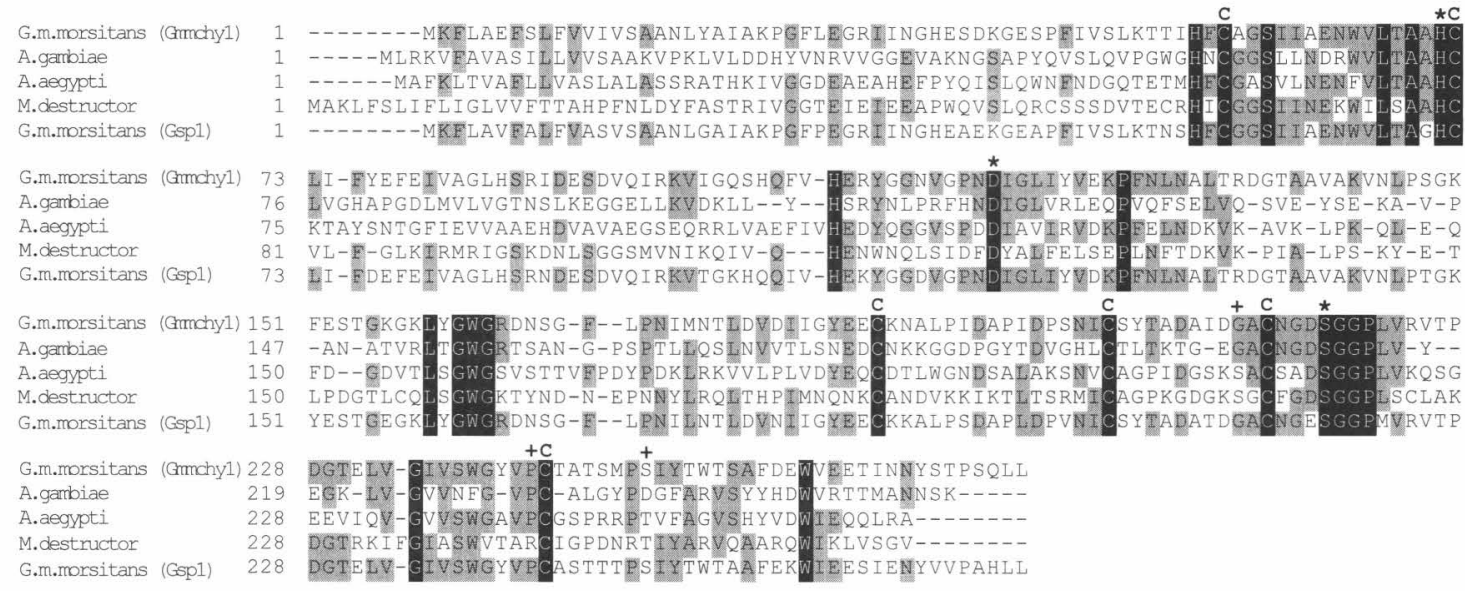

Fig. 1. (A) Multiple alignment of the amino acid sequences of trypsins. G. m. morsitans Gmmtry1 (this study), A. gambiae Antry1 (Z22930), A. aegypti Aaeg 5-5 G1 (X64363), Mayetiola destructor MDP3A (AY 596476), G. m. morsitans Gsp2 (AF252869). The catalytic triad (H/D/S) is indicated by asterisks on top of the sequences. The putative activation cleavage site is marked with an arrow. Conserved cysteines are indicated with $(\mathrm{C})$ on top of the sequences. Hyphens represent sequence alignment gaps. Residues conferring trypsin specificity are presented as + .

(B) Multiple alignment of the amino acid sequences of several chymotrypsins. G. m. morsitans Gmmchy1 (this study), A. gambiae Anchym1 (Z18887), A. aegypti AeCHYM (X64363), M. destructor MDP 2B, (A Y596479), G. m. morsitans Gsp1 (AF252868). The catalytic triad (H/D/S) is indicated by asterisks on top of the sequences. Conserved cysteines are marked with (C) on top of the sequences. Hyphens represent sequence alignment gaps. Residues conferring chymotrypsin specificity are presented as + .

diluted in PBS from the $100 \mathrm{mM}$ stock in dimethylsulfoxide and mixed with a sample equivalent of $1 / 10$ a midgut. Assays were done at $25^{\circ} \mathrm{C}$ in a SPECTRA MAX250 spectrophotometric plate reader set at $405 \mathrm{~nm}$ (Molecular Devices).

\section{RESULTS}

\section{Isolation and characterization of EST clones} encoding proteases

The EST gene discovery project from adult tsetse flies reported 18 cDNAs that 
code for serine proteases in the midgut (Table 1) (Lehane et al., 2003). By Blast search, Gmm3354, Gmm3108, Gmm3359, Gmm2697 and Gmm3340 showed high homology with Glossina trypsin-like serine protease precursor (Yan et al., 2001; acc. no. AAF91346) and Gmm3330, Gmm 3362 and Gmm3230 showed similarity with Glossina chymotrypsin-like serine protease precursor (Yan et al., 2001; acc. no. AAF91345). Gmm3293 showed strong similarity with Drosophila pseudoobscura trypsin-like serine protease (acc. no. XM_ 001361526). Gmm2574 is identical to GmPro3, a serine protease gene previously reported by Hao and Aksoy (2002). Gmm 7528 showed a high homology with GRAAL, a multi-domain serine protease with an immune function from Drosophila (Munier et al., 2004).

Analysis by SignalP indicated the presence of putative signal peptide sequences from Gmm3293, Gmm3216, Gmm3362 and Gmm3230. Two of them, Gmm3293 and Gmm3230, were designated as Gmmtry1 (acc. no. EU589384) and Gmmchy1 (acc. no. EU589385), respective1y. Gmmtry 1 showed the highest homology to the Drosophila trypsin-like gene (GA14937-PA) with 63\% identity, whereas Gmmchy1 showed $82 \%$ identity with Glossina chymotrypsin-like gene (AF 252868).

The deduced amino acids sequences were aligned and showed structural similarities with trypsins and chymotrypsins from other insects (Fig. 1 A, B). The highly conserved catalytic triad for trypsins $(\mathrm{H}, \mathrm{D}, \mathrm{S}$ marked with an *) and cleavage site of putative activation peptides (noted with an arrow) are conserved between R and I (Kraut, 1977). Moreover, the $\mathrm{D}$ and $\mathrm{G}$ residues that confer trypsin specificity (represented +in Fig. 2A) are also present in Gmmtryl (Stroud et al., 1974; Yan et al., 2001). Whereas, the amino acid residues involved in chymotrypsin specificity (noted by + ) are present in Gmmchyl (Vizioli et al., 2001). The $\mathrm{G}$ residue in the substrate

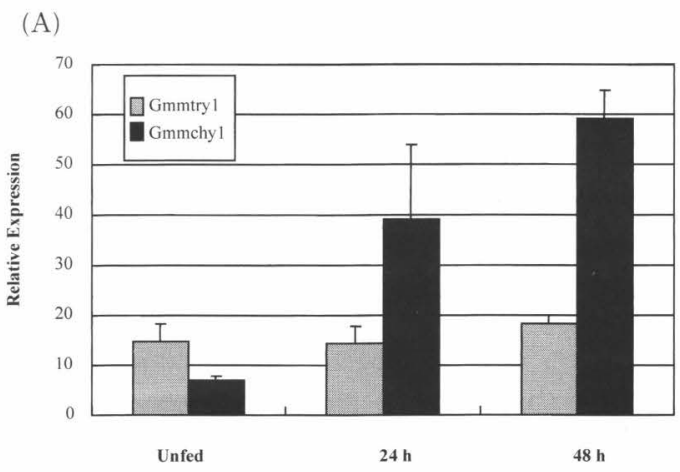

(B)

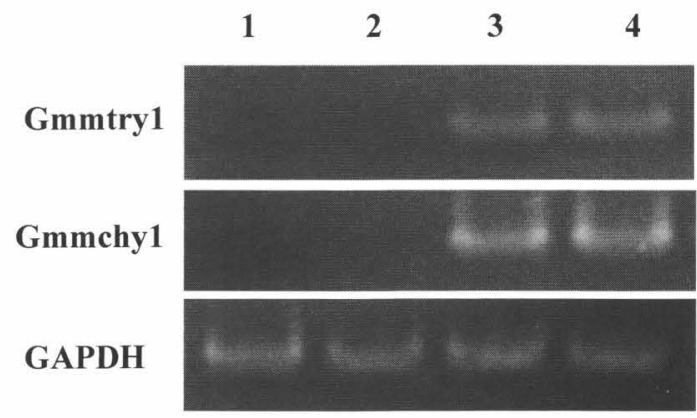

Fig. 2. (A) Gmmtry1 and Gmmchy1 transcription during blood meal digestion. Real-time quantitative PCR was performed on RNAs isolated at the designated times after feeding (unfed, $24 \mathrm{~h}, 48 \mathrm{~h}$ ) from male midguts. Gray bar indicates Gmmtry1; Black bar indicates Gmmchy1. Results are presented with bars indicating the mean \pm standard deviation. The GAPDH gene was used as an internal control.

(B) Expression of Gmmtry1 and Gmmchy1 in the fat body and different parts of the midgut by RT-PCR. The male midgut was divided into 3 parts. Lane 1; Fat body, Lane 2; Anterior midgut containing the proventriculus (blood free portion), Lane 3; Mid midgut (portion containing red undigested blood), Lane 4; Posterior midgut (portion containing blackish digested blood). GAPDH gene was used as an internal control.

binding pocket is replaced by $\mathrm{S}$ at position 250, which is characteristic of chymotrypsins (Kraut, 1977). All sequences contain six spatially conserved cysteine residues that may form three disulfide bridges (marked by C).

Expression after blood feeding and location 


\section{of Gmmtry1 and Gmmchy1}

Total RNA was isolated at $24 \mathrm{~h}$ and $48 \mathrm{~h}$ following a blood meal and analyzed by real-time quantitative PCR for Gmmtry1 and Gmmchy1 genes. Expression of Gmmtry 1 mRNA was stable at low levels until $48 \mathrm{~h}$ post blood meal (PBM), while Gmmchy1 was slightly detectable in unfed midguts, and dramatically increased at 24 $\mathrm{h}$ and $48 \mathrm{~h}$ PBM (Fig. 2A). A previous study showed that Gsp2 and Gsp1 genes are expressed at high levels in the teneral tsetse fly and their expression levels only vary less than $20 \%$ by $48 \mathrm{~h}$ PBM (Yan et al., 2001). To further confirm the tissue specific distribution and location of transcription in the midgut, semi-quantitative RT-PCR was conducted on RNA from specific parts of the midgut and the fat body (Fig. 2B). Midguts were divided into three part: the anterior midgut including the proventriculus (blood free region), mid midgut (the rest of the center region) and posterior midgut (blackened digested blood present). Both Gmmtry1 and Gmmchy1 mRNA was completely absent in the fat body and anterior midgut. In contrast, both genes were expressed at high levels in the mid midgut and posterior midgut. Hao and Aksoy (2002) reported that putative serine protease, GmPro3, is preferentially expressed in the proventriculus.

Trypsin and chymotrypsin enzyme assays in the midgut

The expression profiles of trypsin and chymotrypsin at the protein level were studied by assays for trypsin and chymotrypsin activity in the midgut. Assays followed the methods previously reported by Ramalho-Ortigao et al., (2003). Individual midguts were dissected 6, 24, 48 and $72 \mathrm{~h}$ PBM. The equivalent of $1 / 10$ of a single midgut was incubated with BApNA or AAPF substrates and the colorimetric formation measured after 30 min at $405 \mathrm{~nm}$. As shown in Fig. 3A, trypsin activity was detectable at very low levels even in the unfed midgut. The
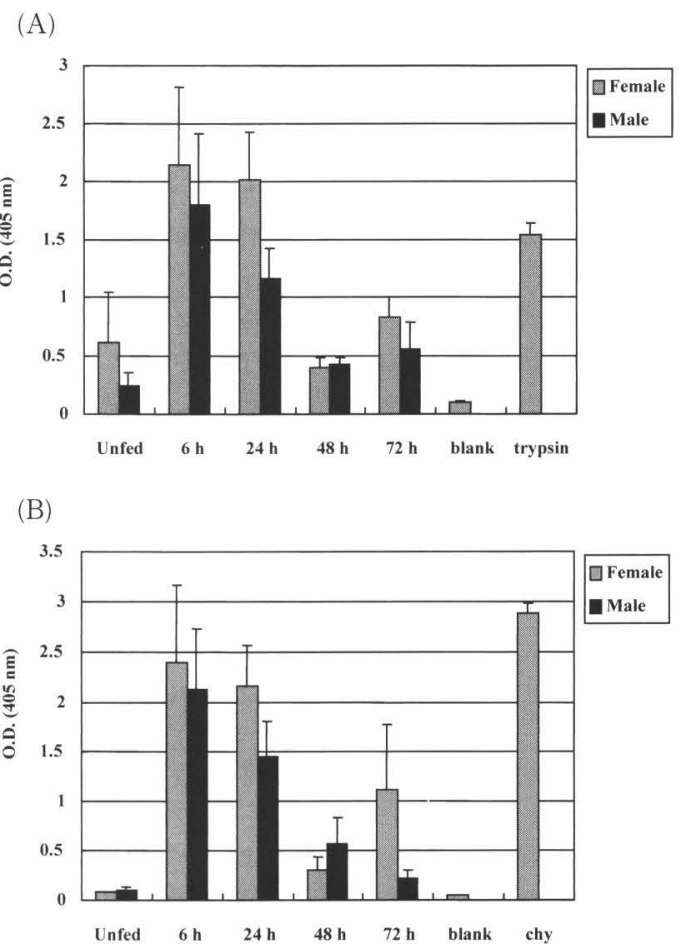

Fig. 3. Glossina morsitans morsitans midgut trypsin and chymotrypsin activity. (A) Trypsin assays were performed on midguts dissected at various times after a blood meal ( $6 \mathrm{~h}, 24 \mathrm{~h}, 48 \mathrm{~h}$ and $72 \mathrm{~h}$ ) and mixed with 1 mM BApNA. (B) Chymotrypsin assays were carried out with AAPF as a substrate. Measurements were performed at $405 \mathrm{~nm}$ with the end point at $30 \mathrm{~min}$. try and chy in the right lane indicate the positive controls (1 mg/ml).

activity increased sharply at $6 \mathrm{~h}$ PBM followed by a reduction at $48 \mathrm{~h}$ PBM. At $72 \mathrm{~h}$ PBM, the activity returned to slightly higher levels. Similarly, chymotrypsin activity was also evaluated (Fig. 3B). Chymotrypsin activity was undetectable in the unfed midguts of both females and males. At $6 \mathrm{~h}$ PBM, activity reached a maximum and then gradually decreased until $48 \mathrm{~h}$ PBM. Enzyme activity is relatively higher in midguts from females than males throughout all times except for $48 \mathrm{~h}$ PBM.

Gene expression during parasite establishment 


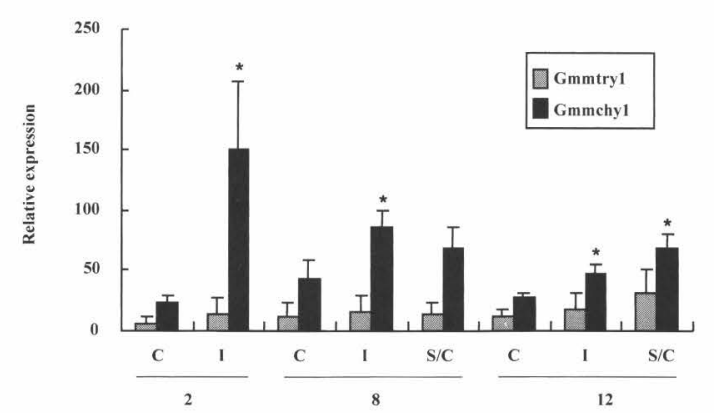

Fig. 4. Analysis of transcriptional levels of Gmmtry1 and Gmmchy1 in trypanosome infected and self cleared midguts of male flies. Results were normalized using the GAPDH gene as an internal control. Gray bar indicates Gmmtry1; Black bar indicates Gmmchy1. C, control flies; I, infected flies; S/ C, self-cleared flies; 2,2 days; 8,8 days; 12,12 days. Range bars represent the standard deviation and asterisks indicate significant differences compared to the controls $(P<$ 0.05).

Transcripts of several genes such as defensin and serine protease increase in the mosquito midgut after infection with Plasmodium (Dimopouloset al., 1997). We examined Gmmtryl and Gmmchyl upregulation in response to parasite infection. To investigate immune responsiveness, flies were fed blood containing the bloodstream form trypanosome $\left(1 \times 10^{6} /\right.$ $\mathrm{ml)}$. Flies were dissected 2, 8, 12 days PBM and midgut parasite infection scored as either infected or self-cleared. The transcription levels of Gmmtry1 and Gmmchy1 on the above days after infection were compared by real-time quantitative PCR (Fig. 4). Although Gmmtryl showed no differences in transcription levels at all times assayed, the expression level of Gmmchyl was significantly higher in infected midguts than the controls at all times tested $(P<0.05)$. Up-regulation of Gmmchy 1 was statistically significant in day 2, 8, 12 infected midguts compared against the controls.

\section{Discussion}

We identified and characterized puta- tive trypsin and chymotrypsin genes from the tsetse fly G. m. morsitans. A total of 18 trypsin and chymotrypsin-like genes were identified from an EST library. Gmmtryl and Gmmchy1 were subjected to further characterization and a high similarity to other insects such as mosquito and sand fly were observed. Glossina serine protease genes Gsp1 (chymotrypsin-like gene) and Gsp2 (trypsin-like gene) are expressed at high levels in the teneral tsetse fly and remain stable until $48 \mathrm{~h}$ PBM suggesting post translational regulation. Both Gsp1 and Gsp2 are encoded by one or possibly two loci in the tsetse genome (Yan et al., 2001). Gmmtry 1 mRNA is constitutively expressed upon blood digestion during the digestive cycle. On the other hand, Gmmchy1 transcripts rapidly increased in response to a blood meal. Although the transcriptional induction of attacin and defensin as well as nitric oxide synthase (NOS) occurs in the preventriculus in response to an immune challenge (Hao et al., 2003), expression of Gmmtry1 and Gmmchy1 was observed mainly in the posterior midgut (Fig. 3B). The trypsin activities of Gsp1 and the chymotrypsin activities of Gsp2 reached peak levels approximately $24 \mathrm{~h}$ after bloodmeal, but the functions for parasite infections were not investigated (Yan et al., 2001). Gmmtry1 mRNA was constitutively expressed at a low levels after blood digestion and enzyme activity of trypsin increased suggesting other multiple trypsins were involved with digestion during the bloodmeal process.

Insect vector midgut proteases may have a direct effect on the parasitic infection and be regulated by the presence of the parasite. Borovsky and Schlein (1987) investigated the possible role of trypsin and chymotrypsin proteases in determining susceptibility of sand flies to certain species of Leishmania. In Phlebotomus papatasi, a specific component of the trypsin-like activity presents the survival of Leishmania donovanni (Borovsky and Schlein, 1987). In Aedes aegypti, midgut 
trypsin has been shown to activate Plasmodium chitinase to traverse the peritrophic membrane (Shahabuddin et al., 1996; Vinetz et al., 2000). In addition, midgut trypsin expression is affected by infection with Plasmodium in Anopheles gambiae (Bonnet et al., 2001). Real-time quantitative PCR indicated significant differences in Gmmchy1 expression between control and infected midguts. Because transcription of Gmmchy1 was upregulated as parasites transformed to the procyclic form in the midgut from 2 to 8 days after an infectious meal, it is possible that Gmmchy1 is induced in the presence of trypanosome infections. No significant differences were observed in Gmmchyl expression between infected and self-cleared after an infectious meal. A possible explanation is that trypanosome debris may remain in the midgut and cause continuous induction of Gmmchy1. The mosquito trypsin-like serine protease, $S p 22 D$, consisting of a chitin binding domain, lowdensity lipoprotein receptor and scavenger receptor cysteine-rich domains also shows an immune response (Danielli et al., 2000). It remains to be seen if the increase in Gmmchy1 transcripts in infected midguts simply reflects the stimulation of the digestion process by the presence of parasites in the blood meal.

Trypanosomes expressing procyclin become visible approximately $3 \mathrm{~h}$ PBM (Gibson and Bailey, 2003). During this stage, the surface glycoproteins of the procyclic forms of $T$. brucei brucei are clipped off by proteolytic enzymes in the midgut of the tsetse fly (Acosta-Serrano et al., 2001; Liniger et al., 2003; Liniger et al., 2004). Therefore, tsetse fly trypsin and chymotrypsin likely trim the glycoproteins from the surface of the trypanosome and play a key role in mechanisms of midgut defense against parasites. Trypsins are also able to kill transitional stage Leishmania amastigotes in the midgut of $P$. papatasi (Pimenta et al., 1997). High concentrations of trypsin enzymes result in complete lysis of trypanosomes (Imbuga et al., 1992). These studies predicted that trypsin directly eliminates the parasites by proteolytic activity in the midgut. Alternatively, the inhibition of parasite development and survival by trypsin and chymotrypsin may reflect a disturbance in the parasites surface coat rather than directly killing the parasites.

Because a large loss of developing parasites occurs predominantly during early establishment in the midgut (Gibson and Bailey, 2003), only a limited number of tsetse flies are able to transmit trypanosome parasites. Several tsetse fly immune factors, including midgut proteases (Imbuga et al., 1992; Osir et al., 1995), lectins (Maudlin and Welburn, 1987), antimicrobial peptides (Boulanger et al., 2002; Hao et al., 2001; Hu and Aksoy, 2005; Hu and Aksoy, 2006), reactive oxygen intermediates (Hao et al., 2003) and antioxidants (MacLeod et al., 2007) are known to respond to establishment of parasites in the midgut.

Although the specific mechanisms of tsetse fly trypsin and chymotrypsin remain to be elucidated, these proteases may cause surface disturbances of parasites in the early stages of infection. Our gene expression analysis suggests that Gmmtry1 might not be involved with parasite elimination. A possible explanation may be that a certain trypsin and/or chymotrypsin in the tsetse serine protease gene family contributes to cleavage of the surface proteins of the procyclic form and facilitate subsequent attack by the antitrypanosomal factors described above.

Further research is needed on the direct effect of trypsin and chymotrypsin on the parasite membrane as well as on the expression analysis in response to Gramnegative and Gram-positive bacteria. Recently para-genetic transformation by bacterial symbionts has been developed (Aksoy, 2003; Rio et al., 2004). Using this technique, expression of recombinant proteins may reveal their roles in Trypanosoma survival in the tsetse fly midgut. 


\section{ACKNOWLEDGEMENTS}

We thank Dr. Hiromitsu Tanaka for his technical support. This work was supported by a grant from the Research Fellowships of the Japan Society for the Promotion of Science.

\section{REFERENCES}

Abubakar, L. U., Bulimo, W. D., Mulaa, F. J. and Osir, E. O. 2006. Molecular characterization of a tsetse fly midgut proteolytic lectin that mediates differentiation of African trypanosomes. Insect. Biochem. Mol. Biol., 36: 344-352.

Acosta-Serrano, A., Vassella, E., Liniger, M., Kunz Renggli, C., Brun, R., Roditi, I. and Englund, P. T. 2001. The surface coat of procyclic Trypanosoma brucei: programmed expression and proteolytic cleavage of procyclin in the tsetse fly. Proc. Natl. Acad. Sci. USA, 98: 1513-1518.

Aksoy, S. 2003. Control of tsetse flies and trypanosomes using molecular genetics. Vet. Parasitol., 115: 125-145.

Aksoy, S., Gibson, W. C. and Lehane, M. J. 2003. Interactions between tsetse and trypanosomes with implications for the control of trypanosomiasis. $A d v$. Parasitol., 53: 1-83.

Bonnet, S., Prevot, G., Jacques, J. C., Boudin, C. and Bourgouin, C. 2001. Transcripts of the malaria vector Anopheles gambiae that are differentially regulated in the midgut upon exposure to invasive stages of Plasmodium falciparum. Cell. Microbiol., 3: 449-458.

Borovsky, D. and Schlein, Y. 1987. Trypsin and chymotrypsin-like enzymes of the sandfly Phlebotomus papatasi infected with Leishmania and their possible role in vector competence. Med. Vet. Entomol., 1: 235-242.

Boulanger, N., Munks, R. J., Hamilton, J. V., Vovelle, F., Brun, R., Lehane, M. J. and Bulet, P. 2002. Epithelial innate immunity. A novel antimicrobial peptide with antiparasitic activity in the bloodsucking insect Stomoxys calcitrans. J. Biol. Chem., 277: 49921-49926.

Danielli, A., Loukeris, T. G., Lagueux, M., Muller, H. M., Richman, A. and Kafatos, F. C. 2000. A modular chitin-binding protease associated with hemocytes and hemolymph in the mosquito Anopheles gambiae. Proc. Natl. Acad. Sci. USA, 97: 7136-7141. Dimopoulos, G., Richman, A., Muller, H. M. and Kafatos, F. C. 1997. Molecular immune responses of the mosquito Anopheles gambiae to bacteria and malaria parasites. Proc. Natl. Acad. Sci. USA, 94: 11508-11513.

Gibson, W. and Bailey, M. 2003. The development of Trypanosoma brucei within the tsetse fly midgut observed using green fluorescent trypanosomes. Kinetoplastid Biol. Dis., 2: 1.

Hao, Z. and Aksoy, S. 2002. Proventriculus-specific cDNAs characterized from the tsetse, Glossina morsitans morsitans. Insect Biochem. Mol. Biol., 32: 1663-1671.

Hao, Z., Kasumba, I. and Aksoy, S. 2003. Proventriculus (cardia) plays a crucial role in immunity in tsetse fly (Diptera: Glossinidiae). Insect Biochem. Mol. Biol., 33: 1155-1164.

Hao, Z., Kasumba, I., Lehane, M. J., Gibson, W. C., Kwon, J. and Aksoy, S. 2001. Tsetse immune responses and trypanosome transmission: implications for the development of tsetse-based strategies to reduce trypanosomiasis. Proc. Natl. Acad. Sci. USA, 98: 12648-12653.

$\mathrm{Hu}, \mathrm{C}$. and Aksoy, S. 2006. Innate immune responses regulate trypanosome parasite infection of the tsetse fly Glossina morsitans morsitans. Mol. Microbiol., 60: 1194-1204.

Hu, Y. and Aksoy, S. 2005. An antimicrobial peptide with trypanocidal activity characterized from Glossina morsitans morsitans. Insect Biochem. Mol. Biol., 35: 105-115.

Imbuga, M. O., Osir, E. O., Labongo, V. L., Darji, N. and Otieno, L. H. 1992. Studies on tsetse midgut factors that induce differentiation of blood-stream Trypanosoma brucei brucei in vitro. Parasitol. Res., 78: 10-15.

Kraut, J. 1977. Serine proteases: structure and mechanism of catalysis. Annu. Rev. Biochem., 46: 331358.

Lehane, M. J., Aksoy, S., Gibson, W., Kerhornou, A., Berriman, M., Hamilton, J., Soares, M. B., Bonaldo, M. F., Lehane, S. and Hall, N. 2003. Adult midgut expressed sequence tags from the tsetse fly Glossina morsitans morsitans and expression analysis of putative immune response genes. Genome. Biol., 4: R63.

Liniger, M., Acosta-Serrano, A., Van Den Abbeele, J., Kunz Renggli, C., Brun, R., Englund, P. T. and Roditi I. 2003. Cleavage of trypanosome surface 
glycoproteins by alkaline trypsin-like enzyme(s) in the midgut of Glossina morsitans. Int. J. Parasitol., 33: 1319-1328.

Liniger, M., Urwyler, S., Studer, E., Oberle, M., Renggli, C. K. and Roditi, I. 2004. Role of the Nterminal domains of EP and GPEET procyclins in membrane targeting and the establishment of midgut infections by Trypanosoma brucei. Mol. Biochem. Parasitol., 137: 247-251.

MacLeod, E. T., Maudlin, I., Darby, A. C. and Welburn, S. C. 2007. Antioxidants promote establishment of trypanosome infections in tsetse. Parasitology, 134: 827-831.

Maudlin, I. and Ellis, D. S. 1985. Association between intracellular rickettsial-like infections of midgut cells and susceptibility to try panosome infection in Glossina spp. Z. Parasitenkd., 71: 683-687.

Maudlin, I. and Welburn, S. C. 1987. Lectin mediated establishment of midgut infections of Trypanosoma congolense and Trypanosoma brucei in Glossina morsitans. Trop. Med. Parasitol., 38: 167-170.

Moloo, S. K. 1971. An artificial feeding technique for Glossina. Parasitology, 63: 507-512.

Moloo, S. K., Kabata, J. M. and Sabwa, C. L. 1994. A study on the maturation of procyclic Trypanosoma brucei brucei in Glossina morsitans centralis and $G$. brevipalpis. Med. Vet. Entomol., 8: 369-374.

Moloo, S. K., Sabwa, C. L. and Kabata, J. M. 1992. Vector competence of Glossina pallidipes and $G$. morsitans centralis for Trypanosoma vivax, T. congolense and T. b. brucei. Acta Trop., 51: 271-280.

Msangi, A. R., Whitaker, C. J. and Lehane, M. J. 1998. Factors influencing the prevalence of trypanosome infection of Glossina pallidipes on the Ruvu flood plain of Eastern Tanzania. Acta Trop., 70: 143-155.

Munier, A. I., Medzhitov, R., Janeway, C. A., Jr., Doucet, D., Capovilla, M. and Lagueux, M. 2004. graal: a Drosophila gene coding for several mosaic serine proteases. Insect Biochem. Mol. Biol., 34: 1025-1035.

Osir, E. O., Abubakar, L. and Imbuga, M. O. 1995. Purification and characterization of a midgut lectin-trypsin complex from the tsetse fly Glossina longipennis. Parasitol. Res., 81: 276-281.

Pimenta, P.F., Modi, G. B., Pereira, S. T., Shahabuddin, M. and Sacks, D. L. 1997. A novel role for the peritrophic matrix in protecting Leishmania from the hydrolytic activities of the sand fly midgut. Parasitology, 115: 359-369.

Ramalho-Ortigao, J. M., Kamhawi, S., Rowton, E. D.,
Ribeiro, J. M. and Valenzuela, J. G. 2003. Cloning and characterization of trypsin- and chymotrypsin-like proteases from the midgut of the sand fly vector Phlebotomus papatasi. Insect Biochem. Mol. Biol., 33: 163-171.

Rio, R. V., Hu, Y. and Aksoy, S. 2004. Strategies of the home-team: symbioses exploited for vector-borne disease control. Trends Microbiol., 12: 325-336.

Ruepp, S., Furger, A., Kurath, U., Renggli, C. K., Hemphill, A., Brun, R. and Roditi, I. 1997. Survival of Trypanosoma brucei in the tsetse fly is enhanced by the expression of specific forms of procyclin. $J$. Cell Biol., 137: 1369-1379.

Shahabuddin, M., Lemos, F. J., Kaslow, D. C. and Jacobs-Lorena, M. 1996. Antibody-mediated inhibition of Aedes aegypti midgut trypsins blocks sporogonic development of Plasmodium gallinaceum. Infect. Immun., 64: 739-743.

Stroud, R. M., Kay, L. M. and Dickerson, R. E. 1974. The structure of bovine trypsin: electron density maps of the inhibited enzyme at 5 Angstrom and at 2-7 Angstron resolution. J. Mol. Biol., 83: 185208.

Vickerman, K., Tetley, L., Hendry, K. A. and Turner, C. M. 1988. Biology of African trypanosomes in the tsetse fly. Biol. Cell., 64: 109-119.

Vinetz, J. M., Valenzuela, J. G., Specht, C. A., Aravind, L., Langer, R. C., Ribeiro, J. M. and Kaslow, D. C. 2000. Chitinases of the avian malaria parasite Plasmodium gallinaceum, a class of enzymes necessary for parasite invasion of the mosquito midgut. $J$. Biol. Chem., 275: 10331-10341.

Vizioli, J., Catteruccia, F., della Torre, A., Reckmann, I. and Muller, H. M. 2001. Blood digestion in the malaria mosquito Anopheles gambiae: molecular cloning and biochemical characterization of two inducible chymotrypsins. Eur. J. Biochem., 268: 4027-4035.

Welburn, S. C. and Maudlin, I. 1999. Tsetse-trypanosome interactions: rites of passage. Parasitol. Today, 15: 399-403.

WHO. 2001. Scientific Working Group on African Try panosomiasis (Sleeping Sickness). WHO/Tropical Disease Research Unit, Geneva.

Yan, J., Cheng, Q., Li, C. B. and Aksoy, S. 2001. Molecular characterization of two serine proteases expressed in gut tissue of the African trypanosome vector, Glossina morsitans morsitans. Insect Mol. Biol., 10: 47-56. 